\title{
Effect of Community Characteristics on Familial Clustering of End-Stage Renal Disease
}

\author{
Eun-Young Song ${ }^{a}$ William M. McClellanc Ann McClellan ${ }^{c}$ Rajyalakshmi Gadi ${ }^{b}$ \\ Alexander C. Hadley ${ }^{b}$ Jenna Krisher ${ }^{d}$ Margo Clay ${ }^{d}$ Barry I. Freedman ${ }^{b}$ \\ a Public Health Sciences and ${ }^{\mathrm{b}}$ Internal Medicine, Wake Forest University School of Medicine, Winston-Salem, N.C., \\ 'Rollins School of Public Health, Emory University, Atlanta, Ga., and d ESRD Network 6, Raleigh, N.C., USA
}

\section{Key Words}

End-stage renal disease - Environment/neighborhood •

Familial aggregation $\cdot$ Geocode $\cdot$ Socioeconomic status

\begin{abstract}
Background: Lower socioeconomic status is generally associated with an increased risk of end-stage renal disease (ESRD). The relationship between community characteristics reflecting socioeconomic status and familial aggregation of common forms of ESRD has not been studied. Methods: Demographic data and family history of ESRD were collected from 23,880 incident dialysis patients in ESRD Network 6 between 1995 and 2003. Addresses were geocoded and linked to the 2000 census 5 -digit zip code-level database that includes community demographic, social and economic characteristics. Clustering of patients having a family history of ESRD at the community level was accounted for using a generalized estimating equations (GEE) model. Multivariate analysis estimated associations between family history of ESRD and community-level characteristics. Results: Twentythree percent of patients reported a family history of ESRD. After adjusting for individual demographic characteristics, multivariate analyses failed to reveal statistically significant relationships between a family history of ESRD and indica-
\end{abstract}

\section{KARGER}

๑ 2009 S. Karger AG, Basel

Fax +41613061234 E-Mail karger@karger.ch www.karger.com www.karger.com/ajn tors of community socioeconomic status such as median household income, percentage high school graduates, percentage vacant housing units or ethnic composition. Conclusions: Although select community measures of lower socioeconomic status may contribute to the familial clustering of ESRD, non-socioeconomic factors, potentially inherited, appear to be more important contributors to familial aggregation of the common forms of ESRD.

Copyright $\odot 2009$ S. Karger AG, Basel

\section{Introduction}

Lack of access to adequate medical care and lower socioeconomic status (SES) are associated with an increased risk of developing chronic kidney disease (CKD) [1]. Familial aggregation of kidney failure has long been recognized in common complex forms of kidney disease, including end-stage renal disease (ESRD) attributed to chronic glomerular disease, diabetes mellitus and hypertension-associated nephropathy [2-4]. The role of environmental exposures, SES, and inherited factors in causation of ESRD remains unclear in these complex kidney disorders. It is important to address this question, as prevention of CKD can be optimized by un-

Barry I. Freedman, MD

Section of Nephrology, Wake Forest University School of Medicine

Medical Center Boulevard

Winston-Salem, NC 27157-1053 (USA)

Tel. +1 336716 6192, Fax +1 336716 4318, E-Mail bfreedma@wfubmc.edu 


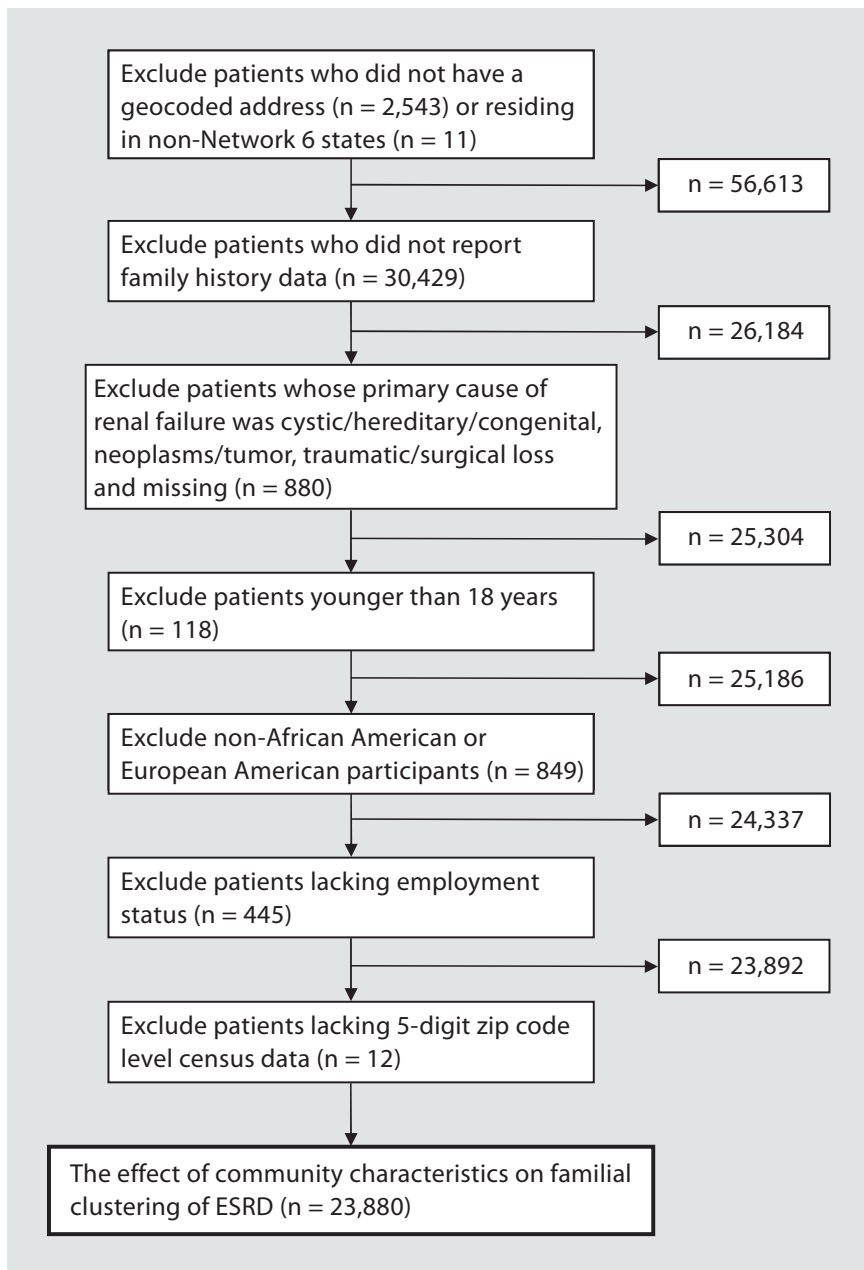

Fig. 1. Network 6 family history of ESRD study $(\mathrm{n}=59,167)$.

derstanding the relative roles that environmental effects (e.g., lack of access to physicians and appropriate medications) and inheritance play in nephropathy susceptibility [5].

Genetic factors clearly influence the development of kidney disease as exemplified by the association of the MYH9 gene with focal segmental glomerulosclerosis, human immunodeficiency virus-associated nephropathy, and the disease labeled 'hypertension-associated ESRD' in African-Americans [6-8]. It is estimated that $70 \%$ of all non-diabetic cases of ESRD in African-Americans are directly attributable to variation in this gene. In contrast, the present analyses were performed to assess the role of environmental factors involving neighborhood characteristics that reflect SES in the common familial forms of ESRD.

\section{Methods}

\section{Sample and Data}

Individual-level data were derived from ESRD Network 6 Family History (FH) of ESRD Study participants [3, 4]. The overall dataset included 59,167 incident dialysis patients starting dialysis therapies between January 1, 1995 and December 31, 2003. The standardized data collection instrument included questions on FH of ESRD in 1st-, 2nd-, and 3rd-degree relatives, age, sex, self-reported ethnicity, number of 1st-degree relatives, etiology of CKD from the CMS 2728 Medical Evidence Report form (containing the designated cause of ESRD as reported by treating physicians), employment status and patient address. This voluntary questionnaire was presented to all dialysis providers to be completed by patients initiating renal replacement therapy in ESRD Network 6 dialysis facilities serving the states of North Carolina, South Carolina and Georgia in the southeastern US. To restrict the analysis to common forms of ESRD [typically attributed to chronic glomerular diseases (primary and secondary forms, e.g., lupus nephritis), diabetes mellitus and hypertension-associated nephropathy], we excluded cases with inherited cystic kidney disease, hereditary nephritis, urologic disease, drug-induced nephritis or surgical nephrectomy. ESRD cases with 'unknown' etiologies of kidney disease were included, since many have undiagnosed glomerular and interstitial diseases. The study protocol was approved by the Institutional Review Board at Wake Forest University School of Medicine in Winston-Salem, N.C., USA.

Community-level data were obtained from the 2000 US Census Summary Files 1 and 3 using American Factfinder at http:// factfinder.census.gov [9]. Five-digit zip code-level census data for demographic composition, SES and residential mobility were extracted. The 2000 US Census and the Network 6 data were linked by geocoded address (5-digit zip code).

After exclusion criteria were applied, the final sample size was 23,880 (fig. 1). FH data were not provided by 30,429 patients, most often due to refusal of their dialysis providers (large dialysis organizations) to permit facility staff to administer the voluntary questionnaire to patients. As previously reported, participation rates fell during the later years of the study; however, rates of a positive FH of ESRD did not change over time, suggesting that there was no inherent selection bias among study participants [4]. Incident dialysis patients lacking valid geocoded addresses $(\mathrm{n}=$ $2,543)$ or residing in non-Network 6 states $(n=11)$ were also excluded. Eight hundred and eighty additional patients were excluded because their primary cause of renal failure was: missing; an inherited cystic renal disease; hereditary nephritis; congenital renal failure; neoplasm/tumor, or traumatic/surgical removal of kidneys. Patients less than 18 years of age $(n=118)$ were excluded, as were those with self-reported ethnicity other than AfricanAmerican or European-American $(\mathrm{n}=849)$, and those without recent (prior 6 month) employment status $(n=445)$. Finally, 12 patients were excluded due to lack of census data at the level of 5digit zip code, leaving a total of 23,880 eligible patients.

\section{Measures}

Dependent Variable

The primary outcome 'FH of ESRD' was considered present if a patient reported having a 1st-, 2 nd- or 3rd-degree relative with ESRD. 
Table 1. Community-level variables and definitions: 2000 US census 5-digit zip code-level data

\begin{tabular}{|c|c|}
\hline Variable & Census definition \\
\hline \multicolumn{2}{|l|}{ Socioeconomic indicators } \\
\hline Median household income & Income in 1999, USD \\
\hline Poverty $^{1}$ & $\begin{array}{l}\text { Poverty status in } 1999 \text { - below } \\
\text { poverty level, \% }\end{array}$ \\
\hline Educational attainment & $\begin{array}{l}\text { High school graduates (includes } \\
\text { equivalency) or college degree, } \%\end{array}$ \\
\hline \multicolumn{2}{|l|}{ Residential mobility } \\
\hline Employment status ${ }^{1}$ & $\begin{array}{l}\text { Unemployed civilian population } \\
16 \text { years and over, } \%\end{array}$ \\
\hline Housing tenure & Vacant housing units, $\%$ \\
\hline \multicolumn{2}{|l|}{ Ethnic composition } \\
\hline European-American ${ }^{1}$ & Caucasian, \% \\
\hline African-American & Black or African-American, $\%$ \\
\hline Hispanic $^{1}$ & $\begin{array}{l}\text { Hispanic or Latino (of any } \\
\text { ethnicity), \% }\end{array}$ \\
\hline
\end{tabular}

${ }^{1}$ Not included in the final model building process due to multi-colinearity.

Independent Variables

Individual-Level Variables. Individual demographic characteristics, employment status and primary cause of ESRD were obtained from ESRD Network 6. These characteristics included age at ESRD onset, gender, ethnicity, number of 1st-degree relatives, prior 6 month fulltime employment status (before dialysis initiation), and diabetes as primary cause of renal failure.

Individual Geocoded Address. Full street addresses were available from subjects. Geocoded patient addresses were derived from Network 6 data using ArcGIS 9.2 [10]. The appropriate census 5 digit zip code was identified for each patient's geocoded address and mapped to census 5-digit zip code area. The patient's geocoded 5-digit zip code was used to link ESRD Network 6 individual-level data with census 2000 community-level (5-digit zip code) data.

Community-Level Variables. 'Community' is defined as a '5digit zip code geographic area'. All community-level variables used in the analysis were based on this definition and we use the terms 'community characteristics' and 'census 5-digit zip codelevel data' interchangeably. Community-level characteristics include socioeconomic indicators, residential mobility, and ethnic composition. Table 1 lists and defines these community-level variables. We categorized each community-level variable into quartiles based on its distribution in the 1,652 5-digit zip code communities and treated them as ordinal variables in the analyses.

\section{Statistical Analysis}

Patients within a community are likely to be more similar to one another compared to patients in other communities, since community populations represent intact social groups [11, 12].
Those reporting a relative with ESRD ('positive FH') were modeled using generalized estimating equations (GEE) for correlated binary responses [13] and for generalized linear models with repeated measures using PROC GENMOD with the REPEATED statement. Odds ratios (OR) and 95\% confidence intervals (CI) were adjusted for all other variables in the model. Data were analyzed using Statistical Analysis Software (SAS) version 9.1 (2004).

\section{Results}

\section{Model Building}

Differences in outcome measures were initially modeled among 3 ethnic categories (European-American, African-American, and other). Analyses were limited to European-American and African-American participants since there was limited power to assess differences between 3 ethnic groups due to the small sample of Hispanics, Asians and other ethnic groups.

Several community-level SES variables were significantly correlated with each other. To screen for multicolinearity, all community-level variables were checked using Pearson's correlation coefficients and computing variance inflation factors by regression analysis. After considering the correlation coefficients and variance inflation factors, we excluded community poverty, unemployment, European-American ethnic composition, and Hispanic ethnic composition variables in our final model. The remaining 4 community-level variables were included in the final model: percent African-American ethnic composition, percent high school graduate or higher attained level of education, percent vacant housing units, and median household income (table 1), along with 6 individual-level variables (gender, age at ESRD, ethnicity, number of 1st-degree relatives, 6-month fulltime employment status prior to dialysis and etiology of ESRD).

\section{Description of the Individual and Community \\ Characteristics}

Table 2 lists individual-level characteristics of the study sample. The mean (SD) age at ESRD onset of the 23,880 patients was $60.6(15.2)$ years, $50.3 \%$ were female, $57.2 \%$ African-American, $46.5 \%$ had diabetes, and $15.9 \%$ were employed fulltime prior to ESRD. Their mean (SD) number of 1st-degree family members was 8.0 (5.7), and $23 \%(5,425)$ reported a FH of ESRD. The 35,287 non-participating incident ESRD patients in Network 6 appeared comparable to the cases, as $49.9 \%$ were female, $54.8 \%$ African-American, $41.9 \%$ had diabetes, and age at ESRD was 59.7 years. 
Table 2. Individual-level characteristics of the 23,880 study subjects

\begin{tabular}{lcr}
\hline Variables & Mean & SD \\
\hline Age, years & 60.6 & 15.2 \\
Number of 1st-degree relatives & 8.0 & 5.7 \\
\hline & $\mathrm{n}$ & $\%$ \\
\hline $\begin{array}{l}\text { Gender } \\
\quad \text { Female }\end{array}$ & 12,013 & 50.3 \\
$\quad$ Male & 11,867 & 49.7 \\
Ethnic group & & \\
$\quad$ African-American & 13,658 & 57.2 \\
$\quad$ European-American & 10,222 & 42.8 \\
Fulltime employment & & \\
$\quad$ Yes & 3,791 & 15.9 \\
$\quad$ No & 20,089 & 84.1 \\
Primary cause of ESRD & & \\
$\quad$ Diabetes & 11,105 & 46.5 \\
$\quad$ Other & 12,775 & 53.5 \\
Family history of ESRD & & \\
$\quad$ Yes & 5,425 & 22.7 \\
$\quad$ No & 18,455 & 77.3 \\
\hline
\end{tabular}

Table 3 lists community-level characteristics. In the 1,652 communities, the median African-American composition was $20.9 \%$, median percent below the poverty level $13.4 \%$, median percent with high school or college education $43.5 \%$, median percent vacant housing units $9.8 \%$, and median household income USD 34,765.

\section{Individual and Community Correlates of FH of ESRD}

Five of six individual-level indicators (including age at ESRD, gender, ethnicity, number of 1st-degree family members, and etiology of ESRD) had significant associations with the odds of having a FH of ESRD, while none of the community indicators had significant associations (table 4). Specifically, the predicted odds of having a FH of ESRD were decreased by approximately $1 \%$ for each 1 year increase in age (for those aged 18 or older). The predicted odds of having a FH of ESRD were increased by $4 \%$ for each 1-number increase in number of 1st-degree family members. The predicted odds of a FH of ESRD were approximately $24 \%$ higher for female than male patients. African-American patients, compared to European-American, had 2.3 times higher predicted odds of a FH of ESRD. Patients with diabetes as cause of ESRD, compared to non-diabetic ESRD, had a 1.16-times higher predicted odds of a FH of ESRD. Significant relationships
Table 3. Community-level characteristics in 1,652 zip code communities: 2000 US census 5-digit zip code-level data

\begin{tabular}{|c|c|c|}
\hline Variable & Mean (SD) & Median \\
\hline \multicolumn{3}{|l|}{ Socioeconomic indicators } \\
\hline Median household income, USD & $37,085(12,340)$ & 34,765 \\
\hline Poverty ${ }^{1}, \%$ & $14.6(7.7)$ & 13.4 \\
\hline High school or college education, \% & $43.6(5.0)$ & 43.5 \\
\hline \multicolumn{3}{|l|}{ Residential mobility } \\
\hline Unemployment $^{1}, \%$ & $3.5(2.9)$ & 3.0 \\
\hline Housing tenure (vacant), $\%$ & $12.2(9.7)$ & 9.8 \\
\hline \multicolumn{3}{|l|}{ Ethnic composition } \\
\hline African-American, $\%$ & $26.0(22.2)$ & 20.9 \\
\hline Hispanic ${ }^{1}, \%$ & $3.3(4.6)$ & 1.9 \\
\hline European-American ${ }^{1}, \%$ & $69.5(22.5)$ & 73.5 \\
\hline
\end{tabular}

${ }^{1}$ Not included in the final model building process to avoid multi-colinearity.

were not detected between individual SES and FH of ESRD.

After adjustment for 6 individual demographic characteristics, multivariate analyses revealed no statistically significant relationships between FH of ESRD and community-level characteristics reflecting SES (table 4). The PROC GENMOD and PROC GLIMMIX analyses were also run as sensitivity analyses and results were not different (data not shown). We performed multivariate analyses on FH of ESRD limited to participants reporting either 1st- or 2nd-degree relatives with ESRD. Similar results were obtained (data not shown).

\section{Discussion}

Familial aggregation of the common causes of ESRD has been appreciated worldwide [14]. It is important to determine whether shared environmental risk factors and lower SES are associated with this clustering, as these factors have been strongly linked to sporadic cases of kidney disease. The present analyses used the ESRD Network 6 'FH of ESRD' database to evaluate demographic and clinical information in 23,880 incident ESRD patients residing in the southeastern United States, and excluded cases with Mendelian disorders (polycystic kidney disease and hereditary nephritis) and environmental causes of kidney failure (obstructive uropathy and surgical nephrectomy). These data were linked with publically available geocode information from the year 2000 census 
Table 4. Final multivariate models for family history of ESRD $(\mathrm{n}=23,880)$

\begin{tabular}{|c|c|c|}
\hline Parameter & OR & $95 \% \mathrm{CI}$ \\
\hline \multicolumn{3}{|l|}{ Individual-level characteristics } \\
\hline Age & 0.99 & $0.98-0.99^{*}$ \\
\hline \multicolumn{3}{|l|}{ Gender } \\
\hline Female & 1.24 & $1.16-1.32^{*}$ \\
\hline \multicolumn{3}{|l|}{ Male (reference) } \\
\hline \multicolumn{3}{|l|}{ Ethnicity } \\
\hline African-American & 2.32 & $2.15-2.51^{*}$ \\
\hline \multicolumn{3}{|l|}{ European-American (reference) } \\
\hline Number of 1 st-degree family members & 1.04 & $1.03-1.04^{*}$ \\
\hline \multicolumn{3}{|l|}{ Primary Cause of ESRD } \\
\hline Diabetes & 1.16 & $1.09-1.23^{*}$ \\
\hline \multicolumn{3}{|l|}{ Other (reference) } \\
\hline \multicolumn{3}{|l|}{ Prior 6-month fulltime employment } \\
\hline Yes & 1.05 & $0.96-1.15$ \\
\hline \multicolumn{3}{|c|}{ Part time or no employment (reference) } \\
\hline \multicolumn{3}{|l|}{ Community-level characteristics } \\
\hline \multicolumn{3}{|c|}{ Community racial composition (\% African-American) } \\
\hline Quartile $4(\geq 39.8 \%)$ & 0.94 & $0.82-1.08$ \\
\hline Quartile $3(20.9-39.7 \%)$ & 1.00 & $0.87-1.15$ \\
\hline Quartile $2(7.4-20.8 \%)$ & 1.02 & $0.89-1.17$ \\
\hline \multicolumn{3}{|l|}{ Quartile $1(<7.4 \%)$ (reference) } \\
\hline \multicolumn{3}{|l|}{ High school graduate or college } \\
\hline Quartile $4(\geq 46.5 \%)$ & 1.04 & $0.92-1.18$ \\
\hline Quartile 3 (43.5-46.4\%) & 0.99 & $0.88-1.12$ \\
\hline Quartile $2(40.8-43.4 \%)$ & 1.04 & $0.92-1.18$ \\
\hline \multicolumn{3}{|l|}{ Quartile $1(<40.8 \%)$ (reference) } \\
\hline \multicolumn{3}{|l|}{ Vacant housing units } \\
\hline Quartile $4(\geq 13.8 \%)$ & 1.07 & $0.94-1.23$ \\
\hline Quartile $3(9.8-13.7 \%)$ & 1.12 & $1.00-1.27$ \\
\hline Quartile $2(6.8-9.7 \%)$ & 1.03 & $0.94-1.14$ \\
\hline \multicolumn{3}{|l|}{ Quartile $1(<6.8 \%)$ (reference) } \\
\hline \multicolumn{3}{|l|}{ Median household income (USD) } \\
\hline \multicolumn{3}{|l|}{ Quartile $4(\geq 41,354)$ (reference) } \\
\hline Quartile $3(34,765-41,353)$ & 0.98 & $0.84-1.13$ \\
\hline Quartile $2(29,207-34,764)$ & 1.05 & $0.93-1.19$ \\
\hline Quartile $1(<29,207)$ & 1.03 & $0.92-1.16$ \\
\hline $\begin{array}{l}\text { OR }=\text { Odds ratio; } 95 \% \mathrm{CI}=95 \% \mathrm{c} \\
{ }^{*} \mathrm{p}<0.0001 .\end{array}$ & $\mathrm{ce}$ & \\
\hline
\end{tabular}

5-digit zip code-level database. Among these voluntary study participants, 18,455 denied having relatives with ESRD and 5,425 (22.7\%) reported a FH of ESRD. After adjustment for family size, we demonstrated that incident dialysis patients having a $\mathrm{FH}$ of ESRD did not appear more likely to reside in geocodes reflecting economically disadvantaged regions, relative to those in sporadic cases of ESRD. Individual prior 6-month fulltime employment and community ethnic composition, community median family income and community level of educational attainment did not differ significantly among geocodes for those with, versus without relatives on dialysis. Given the large size of this database and the relatively high frequency of individuals with relatives on dialysis, the lack of significant differences in these parameters suggests that community-level SES and education attainment are not major contributors to familial causes of ESRD, particularly from diseases such as diabetes, hypertension and glomerulonephritis. Interestingly, Ward [1] reported that associations between measures of SES and kidney disease differed based upon etiology of nephropathy. Individuals with diabetes-associated nephropathy had more profound effects from SES than did those with the Mendelian disorder autosomal dominant polycystic kidney disease, with intermediate effects in those with lupus nephritis [1].

There are several important limitations to this report. Our results relied on data from geocoding, not direct information from participants. However, we have previously reported the accuracy of geocode data [15]. In addition, collecting this volume of information directly from nearly 24,000 incident dialysis patients over a 9-year timeframe would be challenging. It remains possible that some participants incorrectly reported their FH or that their relatives later developed ESRD. We contacted a random sampling of African-American participants to confirm the accuracy of their FH. Direct participant contact revealed that $88 \%$ of cases provided the same FH information as was listed in the ESRD Network 6 FH of ESRD database. Another limitation is use of zip code-level census data as proxies for community characteristics. Zip code-level data are often from regions smaller than 'communities', especially in highly populated urban areas. In contrast, zip codes often include multiple communities in less densely populated rural areas. Additional research remains to be performed on the influence of community SES and FH of ESRD using data from smaller geographical units, as they become available. The ESRD Network 6 'FH of ESRD' did not collect information on the prevalence of relatives with CKDs that had not yet progressed to ESRD. Renal REGARDS collected this information in a population-based US sample and revealed that AfricanAmericans with a FH of ESRD more often had eGFR of $<60 \mathrm{ml} / \mathrm{min}$, relative to African-Americans without a FH [16]. Interestingly, this effect was not observed in European-Americans. The potential impact of a lack of CKD data from relatives in our report is unclear. We suspect that families with two (or more) relatives with ESRD are far more likely to have additional relatives with CKD 
based upon the strong heritability of measures of subclinical nephropathy (albuminuria and GFR) in families [17]. Finally, development of ESRD could cause individuals to move to areas characterized by lower SES as a result of an inability to work. There is no a priori reason to suspect that this occurs more often in those with a $\mathrm{FH}$ of ESRD, relative to those with sporadic ESRD.

In summary, familial clustering of the common causes of ESRD does not appear to be substantially impacted by measures of lower SES in communities where patients reside. The lack of strong relationships between familial ESRD and lower SES is in direct contrast to the results reported in sporadic cases of ESRD (those without relatives on dialysis). It is likely that inherited factors have a substantial effect on familial clustering of ESRD in kidney disease attributed to diabetes mellitus, high blood pressure and chronic glomerular disorders. These data make a strong case for contacting all close relatives of in- cident dialysis patients to recommend annual screening for subclinical nephropathy and renal disease risk factors. We may ultimately reduce the burden of ESRD by improving treatment for proteinuria and renal risk factors earlier in members of families at high risk of kidney disease by virtue of having additional affected members.

\section{Acknowledgements}

This work was supported in part by NIH grant RO1 DK 070941 (B.I.F.). The data reported here have been supplied by the Southeastern Kidney Council, ESRD Network 6, while under CMS contract HHSM-500-2006-NW006C. The interpretation and reporting of these data are the responsibility of the author(s) and in no way should be seen as an official policy or interpretation of the Southeastern Kidney Council or the Centers for Medicare and Medicaid Services.

\section{References}

1 Ward MM: Socioeconomic status and the incidence of ESRD. Am J Kidney Dis 2008;51: 563-572.

2 Ferguson RM, Grim CE, Opgenorth TJ: A familial risk of chronic renal failure among blacks on dialysis? J Clin Epidemiol 1988;41: 1189-1196.

3 Freedman BI, Soucie JM, McClellan WM: Family history of end-stage renal disease among incident dialysis patients. J Am Soc Nephrol 1997;8:1942-1945.

4 Freedman BI, Volkova NV, Satko SG, et al: Population-based screening for family history of end-stage renal disease among incident dialysis patients. Am J Nephrol 2005;25: 529-535.

5 Chan MR, Dall AT, Fletcher KE, et al: Outcomes in patients with chronic kidney disease referred late to nephrologists: a metaanalysis. Am J Med 2007;120:1063-1070.
6 Freedman BI, Hicks PI, Bostrom MA, et al: Polymorphisms in the non-muscle myosin heavy chain 9 gene (MYH9) are strongly associated with end-stage renal disease historically attributed to hypertension in African Americans. Kidney Int 2009;75:736-745

7 Kao WH, Klag MJ, Meoni LA, et al: MYH9 is associated with nondiabetic end-stage renal disease in African Americans. Nat Genet 2008;40:1185-1192.

8 Kopp JB, Smith MW, Nelson GW, et al: MYH9 is a major-effect risk gene for focal segmental glomerulosclerosis. Nat Genet 2008;40:1175-1184.

9 US Census Bureau: Census 2000, Summary Files 1 and 3 (SF 1 and 3). 2002.

10 ESRI. ArcGIS 9.2. 2006.

11 Murray DM, Short B: Intraclass correlation among measures related to alcohol use by young adults: estimates, correlates and applications in intervention studies. J Stud Alcohol 1995;56:681-694.

12 Murray DM, Short B: Intraclass correlation among measures related to alcohol use by school aged adolescents: estimates, correlates and applications in intervention studies. J Drug Educ 1996;26:207-230.
13 Zeger SL, Liang KY: Longitudinal data analysis for discrete and continuous outcomes. Biometrics 1986;42:121-130.

14 Satko SG, Sedor JR, Iyengar SK, et al: Familial clustering of chronic kidney disease. Semin Dial 2007;20:229-236.

15 Song EY, Reboussin BA, Foley KL, et al: Selected community characteristics and underage drinking. Subst Use Misuse 2009;44: 179-194.

16 McClellan W, Speckman R, McClure L, et al: Prevalence and characteristics of a family history of end-stage renal disease among adults in the United States population: Reasons for Geographic and Racial Differences in Stroke (REGARDS) renal cohort study. J Am Soc Nephrol 2007;18:1344-1352.

17 Freedman BI, Bostrom M, Daeihagh PD, et al: Genetic factors in diabetic nephropathy. Clin J Am Soc Nephrol 2007;2:1306-1316. 